\title{
Article \\ Multi-Step Preconditioned Newton Methods for Solving Systems of Nonlinear Equations
}

\author{
Fayyaz Ahmad 1,2,3,*, Malik Zaka Ullah ${ }^{1,4}$, Ali Saleh Alshomrani ${ }^{4}$, Shamshad Ahmad ${ }^{5}$, \\ Aisha M. Alqahtani ${ }^{6}$ and L. Alzaben ${ }^{4}$ \\ 1 Dipartimento di Scienza e Alta Tecnologia, Universita dell'Insubria, Via Valleggio 11, Como 22100, Italy; \\ malik.zakaullah@uninsubria.it \\ 2 Departament de Física i Enginyeria Nuclear, Universitat Politècnica de Catalunya, Eduard Maristanu 10, \\ Barcelona 08019, Spain \\ 3 UCERD (Pvt) Ltd, Islamabad, Pakistan \\ 4 Department of Mathematics, King Abdulaziz University, Jeddah 21589, Saudi Arabia; \\ aszalshomrani@kau.edu.sa (A.S.A.); lalzaben@kau.edu.sa (L.A.) \\ 5 Heat and Mass Transfer Technological Center, Technical University of Catalonia, Colom 11, Terrassa 08222, \\ Spain; shamshad@cttc.upc.edu \\ 6 Princess Nourah bint Abdulrahman University Riyadh, Saudi Arabia; Alqahtani@pnu.edu.sa \\ * Correspondence: fayyaz.ahmad@upc.edu; Tel.: +34-63-206-6627
}

\begin{abstract}
The study of different forms of preconditioners for solving a system of nonlinear equations, by using Newton's method, is presented. The preconditioners provide numerical stability and rapid convergence with reasonable computation cost, whenever chosen accurately. Different families of iterative methods can be constructed by using a different kind of preconditioners. The multi-step iterative method consists of a base method and multi-step part. The convergence order of base method is quadratic and each multi-step add an additive factor of one in the previously achieved convergence order. Hence the convergence of order of an $m$-step iterative method is $m+1$. Numerical simulations confirm the claimed convergence order by calculating the computational order of convergence. Finally, the numerical results clearly show the benefit of preconditioning for solving system of nonlinear equations.
\end{abstract}

Keywords: systems of nonlinear equations; nonlinear preconditioners; multi-step iterative methods; Frozen Jacobian

When computing simple roots of a system of nonlinear equations, Newton's method [1-4] is a classical, well studied procedure that offers quadratic convergence, under suitably mild regularity assumptions. Many researchers have proposed higher order efficient iterative method [5-12] for solving system of nonlinear equations. Recently, some authors have constructed multi-step iterative methods [13-15] for solving system of nonlinear equations. The main benefit of multi-step iterative methods is hidden in the multi-step part. Because, the Jacobian factorization information from the base method is utilized in the multi-step part repeatedly to enhance the convergence order at the cost of the solution of lower and upper triangular systems and a single evaluation of the system of nonlinear equations. X. Wu [16] wrote a note on the improvement of Newton's method for systems of nonlinear equations. In his note, the author introduced the idea of nonlinear preconditioners and showed that the improved Newton method enjoyed the quadratic convergence. Jose et al. [17] used the idea of nonlinear preconditioning to improve the Newton method, for solving the system of nonlinear equations with known multiplicities. Aslam et al. [18] proposed iterative methods for solving nonlinear equations with unknown multiplicity with the help of nonlinear preconditioners. In the another article Aslam and his co-researcher [19] proposed a preconditioned double Newton method with quartic convergence order for the solving system of nonlinear equations. What they have proposed is the following. Let

$$
\mathbf{F}(\mathbf{x})=\left[f_{1}(\mathbf{x}), f_{2}(\mathbf{x}), \cdots, f_{n}(\mathbf{x})\right]^{T}=\mathbf{0}
$$


be the system of nonlinear equations and let us suppose that only simple roots are present. Here $\mathbf{x}=\left[x_{1}, x_{2}, \cdots, x_{n}\right]^{T}$. Assume $\mathbf{G}(\mathbf{x})=\left[g_{1}(\mathbf{x}), g_{2}(\mathbf{x}), \cdots, g_{n}(\mathbf{x})\right]^{T}$ is a function which is non-zero everywhere in its definition domain. We define a new function

$$
\mathbf{Q}(\mathbf{x})=\mathbf{G}(\mathbf{x}) \odot \mathbf{F}(\mathbf{x})=\llbracket \mathbf{G}(\mathbf{x}) \rrbracket \mathbf{F}(\mathbf{x})=\llbracket \mathbf{F}(\mathbf{x}) \rrbracket \mathbf{G}(\mathbf{x}),
$$

where $\odot$ is the element-wise multiplication and $\llbracket \cdot \rrbracket$ represent the diagonal matrix, having as main diagonal its argument. The first order Fréchet derivative of (2) can be computed as

$$
\begin{aligned}
\mathbf{Q}^{\prime}(\mathbf{x}) & =\llbracket \mathbf{F}(\mathbf{x}) \rrbracket \mathbf{G}^{\prime}(\mathbf{x})+\llbracket \mathbf{G}(\mathbf{x}) \rrbracket \mathbf{F}^{\prime}(\mathbf{x}) \\
& =\llbracket \mathbf{G}(\mathbf{x}) \rrbracket\left(\mathbf{F}^{\prime}(\mathbf{x})+\llbracket \mathbf{F}(\mathbf{x}) \rrbracket \llbracket \mathbf{G}(\mathbf{x}) \rrbracket^{-1} \mathbf{G}^{\prime}(\mathbf{x})\right) .
\end{aligned}
$$

The application of Newton method to (2) gives

$$
\begin{aligned}
\mathbf{x}_{k+1} & =\mathbf{x}_{k}-\mathbf{Q}^{\prime}\left(\mathbf{x}_{k}\right)^{-1} \mathbf{Q}\left(\mathbf{x}_{k}\right) \\
& =\mathbf{x}_{k}-\left(\mathbf{F}^{\prime}(\mathbf{x})+\llbracket \mathbf{F}(\mathbf{x}) \rrbracket \llbracket \mathbf{G}(\mathbf{x}) \rrbracket^{-1} \mathbf{G}^{\prime}(\mathbf{x})\right)^{-1} \mathbf{F}(\mathbf{x}) .
\end{aligned}
$$

The convergence order of (4) is quadratic, because the considered scheme is the Newton method for solving the preconditioned system of nonlinear equations $\mathbf{Q}(\mathbf{x})=\mathbf{0}$. If we take $\mathbf{G}(\mathbf{x})=\exp (\boldsymbol{\beta} \odot \mathbf{x})$ then (4) can be written as

$$
\mathbf{x}_{k+1}=\mathbf{x}_{k}-\left(\mathbf{F}^{\prime}(\mathbf{x})+\llbracket \boldsymbol{\beta} \odot \mathbf{F}(\mathbf{x}) \rrbracket\right)^{-1} \mathbf{F}(\mathbf{x}),
$$

where $\boldsymbol{\beta}=\left[\beta_{1}, \beta_{2}, \cdots, \beta_{n}\right]^{T}$.

\section{Our proposal}

In this section, we develop some preconditioned iterative methods for solving systems of nonlinear equations. We generalize the idea of preconditioning in such a way that the quadratic convergence will be guaranteed, under the usual regularity requirements. If we replace $\mathbf{G}(\mathbf{x})$ by $\exp (\mathbf{G}(\mathbf{x}))$ in (4), then we obtain

$$
\mathbf{x}_{k+1}=\mathbf{x}_{k}-\left(\mathbf{F}^{\prime}\left(\mathbf{x}_{k}\right)+\llbracket \mathbf{F}\left(\mathbf{x}_{k}\right) \rrbracket \mathbf{G}^{\prime}\left(\mathbf{x}_{k}\right)\right)^{-1} \mathbf{F}\left(\mathbf{x}_{k}\right) .
$$

Notice that $\mathbf{G}^{\prime}(\mathbf{x})$ is a matrix that could be a diagonal matrix, but also a generic dense matrix. We proposed the following generalization of (6)

$$
\mathbf{x}_{k+1}=\mathbf{x}_{k}-\left(\mathbf{F}^{\prime}\left(\mathbf{x}_{k}\right)+M_{1}\left(\mathbf{x}_{k}\right) \llbracket \mathbf{F}\left(\mathbf{x}_{k}\right) \rrbracket M_{2}\left(\mathbf{x}_{k}\right)\right)^{-1} \mathbf{F}\left(\mathbf{x}_{k}\right),
$$

where $M_{1}(\mathbf{x})$ and $M_{2}(\mathbf{x})$ are matrices of size $n$. In the next development, we see that $\llbracket \mathbf{F}(\mathbf{x}) \rrbracket$ is not the only option. Let $p(\mathbf{x})$ be a scalar function and let us define the following preconditioned system of nonlinear equations

$$
\mathbf{Q}(\mathbf{x})=p(\mathbf{x}) \odot \mathbf{F}(\mathbf{x})=\left[p(\mathbf{x}) f_{1}(\mathbf{x}), p(\mathbf{x}) f_{2}(\mathbf{x}), \cdots, p(\mathbf{x}) f_{n}(\mathbf{x})\right]^{T} .
$$

The first order Fréchet derivative of (8) can be computed as

$$
\begin{aligned}
& \mathbf{Q}^{\prime}(\mathbf{x})=p(\mathbf{x}) \odot \mathbf{F}^{\prime}(\mathbf{x})+\mathbf{F}(\mathbf{x}) \nabla p(\mathbf{x})^{T} \\
& \mathbf{Q}^{\prime}(\mathbf{x})=p(\mathbf{x}) \odot\left(\mathbf{F}^{\prime}(\mathbf{x})+\mathbf{F}(\mathbf{x})\left(\frac{\nabla p(\mathbf{x})^{T}}{p(\mathbf{x})}\right)\right)
\end{aligned}
$$


where $p(\mathbf{x}) \odot \mathbf{F}^{\prime}(\mathbf{x})$ means the element-wise product of $p(\mathbf{x})$ with each element of $\mathbf{F}^{\prime}(\mathbf{x})$ and / in $\left(\frac{\nabla p(\mathbf{x})^{T}}{p(\mathbf{x})}\right)$ is element-wise division. The application of the Newton method to (8) gives

$$
\mathbf{x}_{k+1}=\mathbf{x}_{k}-\left(\mathbf{F}^{\prime}\left(\mathbf{x}_{k}\right)+\mathbf{F}\left(\mathbf{x}_{k}\right)\left(\frac{\nabla p\left(\mathbf{x}_{k}\right)^{T}}{p\left(\mathbf{x}_{k}\right)}\right)\right)^{-1} \mathbf{F}\left(\mathbf{x}_{k}\right)
$$

Again, if we replace $p\left(\mathbf{x}_{k}\right)$ by $\exp \left(p\left(\mathbf{x}_{k}\right)\right)$ in (10), then we have

$$
\mathbf{x}_{k+1}=\mathbf{x}_{k}-\left(\mathbf{F}^{\prime}\left(\mathbf{x}_{k}\right)+\mathbf{F}\left(\mathbf{x}_{k}\right) \nabla p\left(\mathbf{x}_{k}\right)^{T}\right)^{-1} \mathbf{F}\left(\mathbf{x}_{k}\right) .
$$

The following generalization of (11) can be obtained

$$
\begin{aligned}
& \mathbf{x}_{k+1}=\mathbf{x}_{k}-\left(\mathbf{F}^{\prime}\left(\mathbf{x}_{k}\right)+\mathbf{F}\left(\mathbf{x}_{k}\right) \mathbf{V}\left(\mathbf{x}_{k}\right)^{T}\right)^{-1} \mathbf{F}\left(\mathbf{x}_{k}\right) \text { and } \\
& \mathbf{x}_{k+1}=\mathbf{x}_{k}-\left(\mathbf{F}^{\prime}\left(\mathbf{x}_{k}\right)+\mathbf{V}\left(\mathbf{x}_{k}\right) \mathbf{F}\left(\mathbf{x}_{k}\right)^{T}\right)^{-1} \mathbf{F}\left(\mathbf{x}_{k}\right)
\end{aligned}
$$

where $\mathbf{V}\left(\mathbf{x}_{k}\right)=\left[v_{1}\left(\mathbf{x}_{k}\right), v_{2}\left(\mathbf{x}_{k}\right), \cdots, v_{n}\left(\mathbf{x}_{k}\right)\right]^{T}$. Other possibilities could be

$$
\begin{aligned}
& \mathbf{x}_{k+1}=\mathbf{x}_{k}-\left(\mathbf{F}^{\prime}\left(\mathbf{x}_{k}\right)+M_{1}\left(\mathbf{x}_{k}\right) \mathbf{F}\left(\mathbf{x}_{k}\right) \mathbf{V}\left(\mathbf{x}_{k}\right)^{T} M_{2}\left(\mathbf{x}_{k}\right)\right)^{-1} \mathbf{F}\left(\mathbf{x}_{k}\right) \text { and } \\
& \mathbf{x}_{k+1}=\mathbf{x}_{k}-\left(\mathbf{F}^{\prime}\left(\mathbf{x}_{k}\right)+M_{1}\left(\mathbf{x}_{k}\right) \mathbf{V}\left(\mathbf{x}_{k}\right) \mathbf{F}\left(\mathbf{x}_{k}\right)^{T} M_{2}\left(\mathbf{x}_{k}\right)\right)^{-1} \mathbf{F}\left(\mathbf{x}_{k}\right) .
\end{aligned}
$$

Further, we write the multi-step version of the proposed generalizations

$$
\begin{gathered}
\text { Base method } \longrightarrow\left\{\begin{array}{l}
\mathbf{x}_{0}=\text { initial guess } \\
\mathbf{A} \boldsymbol{\phi}_{1}=\mathbf{F}\left(\mathbf{x}_{0}\right) \\
\mathbf{x}_{1}=\mathbf{x}_{0}-\boldsymbol{\phi}_{1}
\end{array}\right. \\
\text { Multi-step part } \rightarrow\left\{\begin{array}{l}
\text { for } j=2, m \\
\mathbf{A} \boldsymbol{\phi}_{j}=\mathbf{F}\left(\mathbf{x}_{j-1}\right) \\
\mathbf{x}_{j}=\mathbf{x}_{j-1}-\boldsymbol{\phi}_{j} \\
\text { end } \\
\mathbf{x}_{0}=\mathbf{x}_{m},
\end{array}\right.
\end{gathered}
$$

where

$$
\mathbf{A}=\left\{\begin{array}{l}
\mathbf{F}^{\prime}(\mathbf{x})+M_{1}(\mathbf{x}) \llbracket \mathbf{F}(\mathbf{x}) \rrbracket M_{2}(\mathbf{x}) \\
\mathbf{F}^{\prime}(\mathbf{x})+\mathbf{F}(\mathbf{x}) \mathbf{V}(\mathbf{x})^{T} \\
\mathbf{F}^{\prime}(\mathbf{x})+\mathbf{V}(\mathbf{x}) \mathbf{F}(\mathbf{x})^{T} \\
\mathbf{F}^{\prime}(\mathbf{x})+M_{1}(\mathbf{x}) \mathbf{F}(\mathbf{x}) \mathbf{V}(\mathbf{x})^{T} M_{2}(\mathbf{x}) \\
\mathbf{F}^{\prime}(\mathbf{x})+M_{1}(\mathbf{x}) \mathbf{V}(\mathbf{x}) \mathbf{F}(\mathbf{x})^{T} M_{2}(\mathbf{x})
\end{array}\right.
$$

Iterative method 
The famous Newton multi-step iterative method can be written as

$$
\begin{gathered}
\text { Base method } \longrightarrow\left\{\begin{array}{l}
\mathbf{x}_{0}=\text { initial guess } \\
\mathbf{F}^{\prime}\left(\mathbf{x}_{0}\right) \boldsymbol{\phi}_{1}=\mathbf{F}\left(\mathbf{x}_{0}\right) \\
\mathbf{x}_{1}=\mathbf{x}_{0}-\boldsymbol{\phi}_{1}
\end{array}\right. \\
\text { Multi-step part } \rightarrow\left\{\begin{array}{l}
\text { for } j=2, m \\
\mathbf{F}^{\prime}\left(\mathbf{x}_{0}\right) \boldsymbol{\phi}_{j}=\mathbf{F}\left(\mathbf{x}_{j-1}\right) \\
\mathbf{x}_{j}=\mathbf{x}_{j-1}-\boldsymbol{\phi}_{j} \\
\text { end } \\
\mathbf{x}_{0}=\mathbf{x}_{m} .
\end{array}\right.
\end{gathered}
$$

\section{Convergence Analysis}

In this section we gice in detail the proofs of convergence order of (16) only for $m=2$, while for the case $m \geq 3$ we use mathematical induction.

Theorem 2.1. Let $\mathbf{F}: \Gamma \subseteq \mathbb{R}^{n} \rightarrow \mathbb{R}^{n}$ be sufficiently Frechet differentiable on an open convex neighborhood $\Gamma$ of $\mathbf{x}^{*} \in \mathbb{R}^{n}$ with $\mathbf{F}\left(\mathbf{x}^{*}\right)=0, \operatorname{det}\left(\mathbf{F}^{\prime}\left(\mathbf{x}^{*}\right)\right) \neq 0$, and with well defined quantities $\left\|M_{1}\left(\mathbf{x}_{k}\right)\right\|,\left\|M_{2}\left(\mathbf{x}_{k}\right)\right\|$, $\left\|\mathbf{V}\left(\mathbf{x}_{k}\right)\right\|$. Then the sequence $\left\{\mathbf{x}_{k}\right\}$ generated by (16) converges to $\mathbf{x}^{*}$ with local order of convergence at least three for $m=2$. Furthermore, the following error inequality

$$
\left\|\mathbf{e}_{k+1}|| \leq\right\| \mathbf{L}\left\||| \mathbf{e}_{k}\right\|^{3}
$$

is satisfied, where $\mathbf{e}_{k}=\boldsymbol{x}_{k}-\mathbf{x}^{*}, \mathbf{e}_{k}^{p}=\overbrace{\left(\mathbf{e}_{k}, \mathbf{e}_{k}, \cdots, \mathbf{e}_{k}\right)}^{p \text { times }}, \mathbf{e}_{k}=\left[\left(\mathbf{e}_{k}\right)_{1},\left(\mathbf{e}_{k}\right)_{2}, \cdots,\left(\mathbf{e}_{k}\right)_{n}\right]^{T}$ and

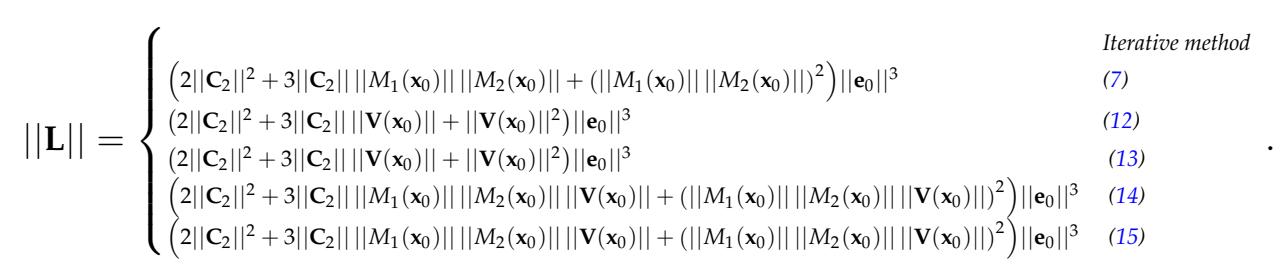

Proof. The $q$ th Frechet derivative of $\mathbf{F}$ at $v \in \mathbb{R}^{n}, q \geq 1$, is the $q$ - linear function $\mathbf{F}^{(q)}(v): \overbrace{\mathbb{R}^{n} \mathbb{R}^{n} \cdots \mathbb{R}^{n}}^{\text {t times }}$ such that $\mathbf{F}^{(q)}(v)\left(u_{1}, u_{2}, \cdots, u_{q}\right) \in \mathbb{R}^{n}$. The Taylor's series expansion of $\mathbf{F}\left(\mathbf{x}_{0}\right)$ around $\mathbf{x}^{*}$ can be written as

$$
\begin{aligned}
\mathbf{F}\left(\mathbf{x}_{0}\right) & =\mathbf{F}\left(\mathbf{x}^{*}+\mathbf{x}_{0}-\mathbf{x}^{*}\right)=\mathbf{F}\left(\mathbf{x}^{*}+\mathbf{e}_{0}\right), \\
& =\mathbf{F}\left(\mathbf{x}^{*}\right)+\mathbf{F}^{\prime}\left(\mathbf{x}^{*}\right) \mathbf{e}_{0}+\frac{1}{2 !} \mathbf{F}^{\prime \prime}\left(\mathbf{x}^{*}\right) \mathbf{e}_{0}^{2}+\frac{1}{3 !} \mathbf{F}^{(3)}\left(\mathbf{x}^{*}\right) \mathbf{e}_{0}^{3}+\cdots, \\
& =\mathbf{F}^{\prime}\left(\mathbf{x}^{*}\right)\left(\mathbf{e}_{0}+\frac{1}{2 !} \mathbf{F}^{\prime}\left(\mathbf{x}^{*}\right)^{-1} \mathbf{F}^{\prime \prime}\left(\mathbf{x}^{*}\right) \mathbf{e}_{0}{ }^{2}+\frac{1}{3 !} \mathbf{F}^{\prime}\left(\mathbf{x}^{*}\right)^{-1} \mathbf{F}^{(3)}\left(\mathbf{x}^{*}\right) \mathbf{e}_{0}{ }^{3}+\cdots\right), \\
& =\mathbf{C}_{1}\left(\mathbf{e}_{0}+\mathbf{C}_{2} \mathbf{e}_{0}{ }^{2}+\mathbf{C}_{3} \mathbf{e}_{0}{ }^{3}+O\left(\mathbf{e}_{0}^{4}\right)\right),
\end{aligned}
$$

where $\mathbf{C}_{1}=\mathbf{F}^{\prime}\left(\mathbf{x}^{*}\right)$ and $\mathbf{C}_{s}=\frac{1}{s !} \mathbf{F}^{\prime}\left(\mathbf{x}^{*}\right)^{-1} \mathbf{F}^{(s)}\left(\mathbf{x}^{*}\right)$ for $s \geq 2$. From (20), we can calculate the Fréchet derivative of $\mathbf{F}$ as

$$
\mathbf{F}^{\prime}\left(\mathbf{x}_{0}\right)=\mathbf{C}_{1}\left(\mathbf{I}+2 \mathbf{C}_{2} \mathbf{e}_{0}+3 \mathbf{C}_{3} \mathbf{e}_{0}^{2}+4 \mathbf{C}_{3} \mathbf{e}_{0}^{3}+O\left(\mathbf{e}_{0}^{4}\right)\right),
$$


where $\mathbf{I}$ is the identity matrix. Before to proceed further, first we compute the norm bounds for

$$
\begin{gathered}
\mathbf{B}(\mathbf{x}) \in\left\{M_{1}(\mathbf{x}) \llbracket \mathbf{F}(\mathbf{x}) \rrbracket M_{2}(\mathbf{x}), \mathbf{F}(\mathbf{x}) \mathbf{V}(\mathbf{x})^{T}, \mathbf{V}(\mathbf{x}) \mathbf{F}(\mathbf{x})^{T}, M_{1}(\mathbf{x}) \mathbf{F}(\mathbf{x}) \mathbf{V}(\mathbf{x})^{T} M_{2}(\mathbf{x}), M_{1}(\mathbf{x}) \mathbf{V}(\mathbf{x}) \mathbf{F}(\mathbf{x})^{T} M_{2}(\mathbf{x})\right\} \\
\mathbf{B}\left(\mathbf{x}_{0}\right)=M_{1}\left(\mathbf{x}_{0}\right) \llbracket \mathbf{F}\left(\mathbf{x}_{0}\right) \rrbracket M_{2}\left(\mathbf{x}_{0}\right) \\
=M_{1}\left(\mathbf{x}_{0}\right) \llbracket \mathbf{C}_{1}\left(\mathbf{e}_{0}+\mathbf{C}_{2} \mathbf{e}_{0}^{2}+O\left(\mathbf{e}_{0}^{3}\right)\right) \rrbracket M_{2}\left(\mathbf{x}_{0}\right) \\
\begin{aligned}
& \mid \mathbf{B}\left(\mathbf{x}_{0}\right) \| \leq\left\|\mathbf{C}_{1}\right\|\left\|M_{1}\left(\mathbf{x}_{0}\right)\right\|\left\|M_{2}\left(\mathbf{x}_{0}\right)\right\|\left\|\mathbf{e}_{0}\right\| \\
& \mathbf{B}\left(\mathbf{x}_{0}\right)=\mathbf{F}\left(\mathbf{x}_{0}\right) \mathbf{V}\left(\mathbf{x}_{0}\right)^{T} \\
&=\mathbf{C}_{1}\left(\mathbf{e}_{0}+\mathbf{C}_{2} \mathbf{e}_{0}^{2}+O\left(\mathbf{e}_{0}^{3}\right)\right) \mathbf{V}\left(\mathbf{x}_{0}\right)^{T} \\
&\left\|\mathbf{B}\left(\mathbf{x}_{0}\right)\right\| \leq\left\|\mathbf{C}_{1}\right\|\left\|\mathbf{V}\left(\mathbf{x}_{0}\right)\right\|\left\|\mathbf{e}_{0}\right\| \\
& \mathbf{B}\left(\mathbf{x}_{0}\right)=\mathbf{V}\left(\mathbf{x}_{0}\right) \mathbf{F}\left(\mathbf{x}_{0}\right)^{T} \\
&=\mathbf{V}\left(\mathbf{x}_{0}\right)\left(\mathbf{C}_{1}\left(\mathbf{e}_{0}+\mathbf{C}_{2} \mathbf{e}_{0}^{2}+O\left(\mathbf{e}_{0}^{3}\right)\right)\right)^{T} \\
&\left\|\mathbf{B}\left(\mathbf{x}_{0}\right)\right\| \leq\left\|\mathbf{C}_{1}\right\|\left\|\mathbf{V}\left(\mathbf{x}_{0}\right)\right\|\left\|\mathbf{e}_{0}\right\| \\
& \mathbf{B}\left(\mathbf{x}_{0}\right)=M_{1}\left(\mathbf{x}_{0}\right) \mathbf{F}\left(\mathbf{x}_{0}\right) \mathbf{V}\left(\mathbf{x}_{0}\right)^{T} M_{2}\left(\mathbf{x}_{0}\right) \\
&=M_{1}\left(\mathbf{x}_{0}\right)\left(\mathbf{C}_{1}\left(\mathbf{e}_{0}+\mathbf{C}_{2} \mathbf{e}_{0}^{2}+O\left(\mathbf{e}_{0}^{3}\right)\right)\right) \mathbf{V}\left(\mathbf{x}_{0}\right)^{T} M_{2}\left(\mathbf{x}_{0}\right) \\
&\left\|\mathbf{B}\left(\mathbf{x}_{0}\right)\right\| \leq \mathbf{C}_{1}\|\| M_{1}\left(\mathbf{x}_{0}\right)\|\| M_{2}\left(\mathbf{x}_{0}\right)\|\| \mathbf{V}\left(\mathbf{x}_{0}\right)\|\| \mathbf{e}_{0} \|
\end{aligned}
\end{gathered}
$$

Notice that all the expressions for $\mathbf{B}(\mathbf{x})$ are of order $\mathbf{e}$ and this is essential in proving the quadratic convergence.

$$
\begin{aligned}
\mathbf{B}\left(\mathbf{x}_{0}\right) & =M_{1}\left(\mathbf{x}_{0}\right) \mathbf{V}\left(\mathbf{x}_{0}\right) \mathbf{F}\left(\mathbf{x}_{0}\right)^{T} M_{2}\left(\mathbf{x}_{0}\right) \\
& =M_{1}\left(\mathbf{x}_{0}\right) \mathbf{V}\left(\mathbf{x}_{0}\right)\left(\mathbf{C}_{1}\left(\mathbf{e}_{0}+\mathbf{C}_{2} \mathbf{e}_{0}^{2}+O\left(\mathbf{e}_{0}^{3}\right)\right)\right)^{T} M_{2}\left(\mathbf{x}_{0}\right) \\
\left\|\mathbf{B}\left(\mathbf{x}_{0}\right)\right\| & \leq\left\|\mathbf{C}_{1}\right\|\left\|M_{1}\left(\mathbf{x}_{0}\right)\right\|\left\|M_{2}\left(\mathbf{x}_{0}\right)\right\|\left\|\mathbf{V}\left(\mathbf{x}_{0}\right)\right\|\left\|\mathbf{e}_{0}\right\| \\
\mathbf{A} & =\mathbf{F}^{\prime}\left(\mathbf{x}_{0}\right)+\mathbf{B}\left(\mathbf{x}_{0}\right) \\
& =\mathbf{C}_{1}\left(\mathbf{I}+2 \mathbf{C}_{2} \mathbf{e}_{0}+\mathbf{C}_{1}^{-1} \mathbf{B}\left(\mathbf{x}_{0}\right)+O\left(\mathbf{e}_{0}^{2}\right)\right) \\
\mathbf{A}^{-1} & =\left(\mathbf{I}-2 \mathbf{C}_{2} \mathbf{e}_{0}-\mathbf{C}_{1}^{-1} \mathbf{B}\left(\mathbf{x}_{0}\right)+O\left(\left(\mathbf{e}_{0}\right)_{i_{1}}\left(\mathbf{e}_{0}\right)_{i_{2}}\right)\right) \mathbf{C}_{1}^{-1}
\end{aligned}
$$

The explanation to use the notation $O\left(\left(\mathbf{e}_{0}\right)_{i_{1}}\left(\mathbf{e}_{0}\right)_{i_{2}}\right)$ is that the quadratic terms are not of the form $\mathbf{e}_{0}^{2}$ because of $\mathbf{B}\left(\mathbf{x}_{0}\right)$. By using (27), we deduce

$$
\begin{aligned}
\mathbf{A}^{-1} \mathbf{F}\left(\mathbf{x}_{0}\right) & =\left(\mathbf{I}-2 \mathbf{C}_{2} \mathbf{e}_{0}-\mathbf{C}_{1}^{-1} \mathbf{B}\left(\mathbf{x}_{0}\right)+O\left(\left(\mathbf{e}_{0}\right)_{i_{1}}\left(\mathbf{e}_{0}\right)_{i_{2}}\right)\right) \mathbf{C}_{1}^{-1} \mathbf{C}_{1}\left(\mathbf{e}_{0}+\mathbf{C}_{2} \mathbf{e}_{0}^{2}+O\left(\mathbf{e}_{0}^{3}\right)\right) \\
& =\mathbf{e}_{0}+\mathbf{C}_{2} \mathbf{e}_{0}^{2}-2 \mathbf{C}_{2} \mathbf{e}_{0}^{2}-\mathbf{C}_{1}^{-1} \mathbf{B}\left(\mathbf{x}_{0}\right) \mathbf{e}_{0}+O\left(\left(\mathbf{e}_{0}\right)_{i_{1}}\left(\mathbf{e}_{0}\right)_{i_{2}}\left(\mathbf{e}_{0}\right)_{i_{3}}\right) \\
\mathbf{e}_{1} & =\mathbf{e}_{0}-\mathbf{e}_{0}+\mathbf{C}_{2} \mathbf{e}_{0}^{2}+\mathbf{C}_{1}^{-1} \mathbf{B}\left(\mathbf{x}_{0}\right) \mathbf{e}_{0}+O\left(\left(\mathbf{e}_{0}\right)_{i_{1}}\left(\mathbf{e}_{0}\right)_{i_{2}}\left(\mathbf{e}_{0}\right)_{i_{3}}\right) \\
\mathbf{e}_{1} & =\mathbf{C}_{2} \mathbf{e}_{0}^{2}+\mathbf{C}_{1}^{-1} \mathbf{B}\left(\mathbf{x}_{0}\right) \mathbf{e}_{0}+O\left(\left(\mathbf{e}_{0}\right)_{i_{1}}\left(\mathbf{e}_{0}\right)_{i_{2}}\left(\mathbf{e}_{0}\right)_{i_{3}}\right)
\end{aligned}
$$


The error equation $\mathbf{e}_{1}$ tells that the order of convergence of base method of (16) is quadratic because $\mathbf{C}_{1}^{-1} \mathbf{B}\left(\mathbf{x}_{0}\right) \mathbf{e}_{0}$ is a second order term in $\mathbf{e}_{0}$. By using (28), (22), (23), (24), (25) and (26), we get

$$
\begin{aligned}
& \mathbf{F}\left(\mathbf{x}_{1}\right)=\mathbf{C}_{1}\left(\mathbf{e}_{1}+O\left(\mathbf{e}_{1}^{2}\right)\right) \\
& =\mathbf{C}_{1}\left(\mathbf{C}_{2} \mathbf{e}_{0}^{2}+\mathbf{C}_{1}^{-1} \mathbf{B}\left(\mathbf{x}_{0}\right) \mathbf{e}_{0}+O\left(\left(\mathbf{e}_{0}\right)_{i_{1}}\left(\mathbf{e}_{0}\right)_{i_{2}}\left(\mathbf{e}_{0}\right)_{i_{3}}\right)\right) \\
& \mathbf{e}_{2}=\mathbf{e}_{1}-\mathbf{A}^{-1} \mathbf{F}\left(\mathbf{x}_{1}\right) \\
& =\mathbf{C}_{2} \mathbf{e}_{0}^{2}+\mathbf{C}_{1}^{-1} \mathbf{B}\left(\mathbf{x}_{0}\right) \mathbf{e}_{0}-\left(\mathbf{I}-2 \mathbf{C}_{2} \mathbf{e}_{0}-\mathbf{C}_{1}^{-1} \mathbf{B}\left(\mathbf{x}_{0}\right)+O\left(\left(\mathbf{e}_{0}\right)_{i_{1}}\left(\mathbf{e}_{0}\right)_{i_{2}}\right)\right)\left(\mathbf{C}_{2} \mathbf{e}_{0}^{2}+\mathbf{C}_{1}^{-1} \mathbf{B}\left(\mathbf{x}_{0}\right) \mathbf{e}_{0}\right) \\
& +O\left(\left(\mathbf{e}_{0}\right)_{i_{1}}\left(\mathbf{e}_{0}\right)_{i_{2}}\left(\mathbf{e}_{0}\right)_{i_{3}}\left(\mathbf{e}_{0}\right)_{i_{4}}\right) \\
& =2 \mathbf{C}_{2}^{2} \mathbf{e}_{0}^{3}+2 \mathbf{C}_{2} \mathbf{e}_{0} \mathbf{C}_{1}^{-1} \mathbf{B}\left(\mathbf{x}_{0}\right) \mathbf{e}_{0}+\mathbf{C}_{1}^{-1} \mathbf{B}\left(\mathbf{x}_{0}\right) \mathbf{C}_{2} \mathbf{e}_{0}^{2}+\mathbf{C}_{1}^{-1} \mathbf{B}\left(\mathbf{x}_{0}\right) \mathbf{C}_{1}^{-1} \mathbf{B}\left(\mathbf{x}_{0}\right) \mathbf{e}_{0}+O\left(\left(\mathbf{e}_{0}\right)_{i_{1}}\left(\mathbf{e}_{0}\right)_{i_{2}}\left(\mathbf{e}_{0}\right)_{i_{3}}\left(\mathbf{e}_{0}\right)_{i_{4}}\right), \\
& \left\|\mathbf{e}_{2}|| \leq 2|| \mathbf{C}_{2}\right\|^{2}|| \mathbf{e}_{0}\left\|^{3}+3\right\| \mathbf{C}_{2}||\left\|\mathbf{C}_{1}\right\|^{-1}|| \mathbf{B}\left(\mathbf{x}_{0}\right)\|\| \mathbf{e}_{0}\left\|^{2}+\right\| \mathbf{C}_{1}\left\|^{-2}\right\| \mathbf{B}\left(\mathbf{x}_{0}\right)\left\|^{2}\right\| \mathbf{e}_{0} \|,
\end{aligned}
$$

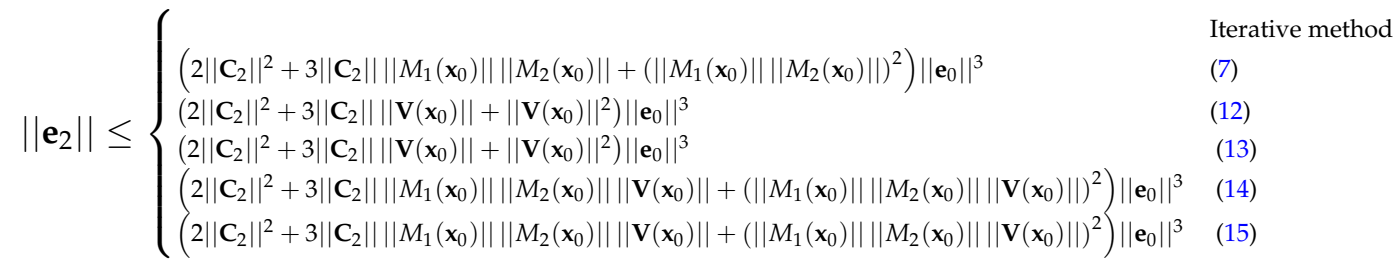

The error inequality (29) completes the proof.

The proof of convergence when $m \geq 3$ can be carried via mathematical induction. Suppose the propose the iterative method (16) has convergence order $s$ when $m=s-1$. The error inequality for $(s-1)$-step iterative method (16) can be written as

$$
\left\|\mathbf{e}_{s-1}\right\| \leq\left\|\mathbf{N}_{1}\right\|\left\|\mathbf{e}_{0}\right\|^{S},
$$

where $\left\|\mathbf{N}_{1}\right\|$ is finite. The error equation for $m=s$ is

$$
\begin{aligned}
\mathbf{e}_{s} & =\mathbf{e}_{s-1}-\mathbf{A}^{-1} \mathbf{F}\left(\mathbf{x}_{s-1}\right) \\
& =\mathbf{e}_{s-1}-\left(\mathbf{I}-2 \mathbf{C}_{2} \mathbf{e}_{0}-\mathbf{C}_{1}^{-1} \mathbf{B}\left(\mathbf{x}_{0}\right)+O\left(\left(\mathbf{e}_{0}\right)_{i_{1}}\left(\mathbf{e}_{0}\right)_{i_{2}}\right)\right) \mathbf{e}_{s-1} \\
\left\|\mathbf{e}_{s}\right\| & \leq 2\left\|\mathbf{C}_{2}\right\|\left\|\mathbf{N}_{1}\right\|\left\|\mathbf{e}_{0}\right\|^{s+1}+\left\|\mathbf{C}_{1}\right\|^{-1}\|\mathbf{B}(\mathbf{x})\|\left\|\mathbf{N}_{1}\right\|\left\|\mathbf{e}_{0}\right\|^{s} \\
\left\|\mathbf{e}_{s}\right\| & \leq\left\|\mathbf{N}_{2}\right\|\left\|\mathbf{e}_{0}\right\|^{s+1},
\end{aligned}
$$

where $\left\|\mathbf{N}_{2}\right\|$ is finite.

\section{Numerical simulations}

Let $\boldsymbol{\alpha}$ be a simple root of system of nonlinear equations $\mathbf{F}(\mathbf{x})=\mathbf{0}$. We adopt the following definition of computational order of convergence

$$
\mathrm{CCO}=\frac{\log \left(\left\|\mathbf{F}\left(\mathbf{x}_{k+1}\right)\right\|_{\infty} /\left\|\mathbf{F}\left(\mathbf{x}_{k}\right)\right\|_{\infty}\right)}{\log \left(\left\|\mathbf{F}\left(\mathbf{x}_{k}\right)\right\|_{\infty} /\left\|\mathbf{F}\left(\mathbf{x}_{k-1}\right)\right\|_{\infty}\right)}
$$

To check the performance of our proposed iterative methods, we solve three problems. 


$$
\begin{aligned}
& \text { Problem } 1=\left\{\begin{array}{l}
x_{i}^{2} x_{i+1}-1=0, \quad i=1,2 \cdots, n-1 \\
x_{n} x_{1}-1=0, \quad i=n
\end{array}\right. \\
& \text { Problem } 2=\left\{\begin{array}{l}
F_{1}(\mathbf{x})=\left(3-0.5 x_{1}\right) x_{1}-2 x_{2}+1 \\
F_{2}(\mathbf{x})=\left(3-0.5 x_{n}\right) x_{n}-2 x_{n-1}+1 \\
F_{i}(\mathbf{x})=\left(3-0.5 x_{i}\right) x_{i}-x_{i-1}+2 x_{i+1}+1, i=2,3 \cdots, n-1
\end{array}\right. \\
& \text { Problem 3 }=\left\{\begin{array}{l}
F_{1}(\mathbf{x})=10 x_{1}+\sin \left(x_{1}+x_{2}\right)-1=0 \\
F_{2}(\mathbf{x})=8 x_{2}-\cos ^{2}\left(x_{3}-x_{2}\right)-1=0 \\
F_{3}(\mathbf{x})=12 x_{3}+\sin \left(x_{3}\right)-1=0
\end{array}\right.
\end{aligned}
$$

Tables 1, 2, and 3 clearly show that the claimed orders of convergence are in agreement with computational orders of convergence for a given number of steps. The simulation times in each Table for all the conducted tests are almost equal. In Table 3, we have used full matrices as preconditioners because the system of nonlinear equations is small. But for a large system of nonlinear equations, it is not recommendable to use full matrices as a preconditioners and reason is clear, since we get a penalty in terms of computational cost. One of the main targets of the present article is to explore different possibilities of preconditioning, by keeping the convergence order. In our analysis, we found that for solving system of nonlinear equations, the iterative method (7) is the most efficient when we use the preconditioning matrices $M_{1}(\mathbf{x})$ and $M_{2}(\mathbf{x})$ as diagonal matrices. It is also observed that the leading constant coefficients of preconditioners should be less one in magnitude to get better accuracy. The proposed iterative methods show better accuracy compared with multi-step Newton method for almost all tests, when considering the previous three model problems.

Table 1. Problem 1: Initial guess: $x_{i}=15 / 10, n=200$, Iter $=5$

\begin{tabular}{ccccc|cc}
\hline \multicolumn{5}{c}{ Method (7) } & \multicolumn{2}{c}{ Method (17) } \\
$M_{1}(\mathbf{x})$ & $M_{2}(\mathbf{x})$ & $m$ & $\|\mathbf{F}(\mathbf{x})\|_{\infty}$ & $\mathrm{CCO}$ & $\|\mathbf{F}(\mathbf{x})\|_{\infty}$ & $\mathrm{CCO}$ \\
\hline $\mathbf{I}$ & $\llbracket-\sin (\mathbf{x}) /(1.1+\cos (\mathbf{x})) \rrbracket$ & 1 & $2.97 e-23$ & 2.0 & $3.20 e-7$ & 1.99 \\
$\mathbf{I}$ & $-\mathbf{I}$ & 2 & $1.95 e-127$ & 4.0 & $4.15 e-28$ & 3.0 \\
$\mathbf{I}$ & $\llbracket-2 \exp (-2 \mathbf{x}) \rrbracket$ & 3 & $7.20 e-89$ & 4.0 & $5.56 e-80$ & 4.0 \\
$\mathbf{I}$ & $\llbracket-\exp (-\mathbf{x}) \rrbracket$ & 4 & $3.21 e-221$ & 5.0 & $3.20 e-185$ & 5.0 \\
\hline
\end{tabular}

\section{Conclusions}

The computational cost of the classical multi-step Newton method and that of the proposed iterative methods are substantially tre same, if we do not use dense preconditioners. Indeed, the iterative method (7) is more effective, when coupled with diagonal preconditioners. The proposed family of iterative methods has the same convergence order as that of Newton multi-step iterative method, with almost the same computational cost. The only assumption on the preconditioners is that they should have finite norms, in their definition domain.

Author contributions: First author established the idea and all other authors contributed equally in the article.

Conflicts of interest: The authors declare no conflict of interest. 
Table 2. Problem 2: Initial guess: $x_{i}=-1, n=100$, Iter $=4$

\begin{tabular}{|c|c|c|c|c|c|c|}
\hline \multicolumn{5}{|c|}{ Method (7) } & \multicolumn{2}{|c|}{ Method (17) } \\
\hline$M_{1}(\mathbf{x})$ & $M_{2}(\mathbf{x})$ & $m$ & $\|\mathbf{F}(\mathbf{x})\|_{\infty}$ & $\mathrm{CCO}$ & $\|\mathbf{F}(\mathbf{x})\|_{\infty}$ & $\mathrm{CCO}$ \\
\hline $\mathbf{I}$ & $\llbracket 1 / 10 \rrbracket$ & 3 & $2.33 e-219$ & 4.0 & $5.92 e-163$ & 4.0 \\
\hline I & $\llbracket 1 / 10 \rrbracket$ & 4 & $4.09 e-511$ & 5.0 & $1.18 e-388$ & 5.0 \\
\hline $\mathbf{I}$ & $\llbracket 1 / 10 \exp (\mathbf{x} / 10) \rrbracket$ & 3 & $4.92 e-214$ & 4.0 & $5.92 e-163$ & 4.0 \\
\hline $\mathbf{I}$ & $\llbracket 1 / 10 \exp (\mathbf{x} / 10) \rrbracket$ & 4 & $3.79 e-499$ & 5.0 & $1.18 e-388$ & 5.0 \\
\hline $\mathbf{I}$ & $\llbracket \cosh (\mathbf{x}) /(10+\sinh (\mathbf{x})) \rrbracket$ & 3 & $3.52 e-264$ & 4.0 & $5.92 e-163$ & 4.0 \\
\hline $\mathbf{I}$ & $\llbracket \cosh (\mathbf{x}) /(10+\sinh (\mathbf{x})) \rrbracket$ & 4 & $2.77 e-614$ & 5.0 & $1.18 e-388$ & 5.0 \\
\hline $\mathbf{I}$ & $\llbracket \cosh (\mathbf{x}) / 10 \rrbracket$ & 3 & $1.97 e-292$ & 4.0 & $5.92 e-163$ & 4.0 \\
\hline $\mathbf{I}$ & $\llbracket \cosh (\mathbf{x}) / 10 \rrbracket$ & 4 & $7.68 e-677$ & 5.0 & $1.18 e-388$ & 5.0 \\
\hline $\mathbf{I}$ & $\llbracket \operatorname{sech}(\mathbf{x}) / 10 \rrbracket$ & 3 & $1.33 e-200$ & 4.0 & $5.92 e-163$ & 4.0 \\
\hline $\mathbf{I}$ & $\llbracket \operatorname{sech}(\mathbf{x}) / 10 \rrbracket$ & 4 & $4.26 e-469$ & 5.0 & $1.18 e-388$ & 5.0 \\
\hline $\mathbf{I}$ & $\llbracket \cos (\mathbf{x}) / 3 \rrbracket$ & 3 & $3.09 e-267$ & 4.0 & $5.92 e-163$ & 4.0 \\
\hline $\mathbf{I}$ & $\llbracket \cos (\mathbf{x}) / 3 \rrbracket$ & 4 & $2.55 e-623$ & 5.0 & $1.18 e-388$ & 5.0 \\
\hline I & $\llbracket\left(1+x^{3}\right) / 3 \rrbracket$ & 3 & $5.71 e-187$ & 4.0 & $5.92 e-163$ & 4.0 \\
\hline I & $\llbracket\left(1+x^{3}\right) / 3 \rrbracket$ & 4 & $4.09 e-443$ & 5.0 & $1.18 e-388$ & 5.0 \\
\hline I & $\llbracket \sinh \left(\mathbf{x}^{2}\right) / 10 \rrbracket$ & 3 & $7.21 e-217$ & 4.0 & $5.92 e-163$ & 4.0 \\
\hline I & $\llbracket \sinh \left(\mathbf{x}^{2}\right) / 10 \rrbracket$ & 4 & $4.16 e-462$ & 5.0 & $1.18 e-388$ & 5.0 \\
\hline $\mathbf{I}$ & $\llbracket \mathrm{x}^{2} / 10 \rrbracket$ & 3 & $8.41 e-204$ & 4.0 & $5.92 e-163$ & 4.0 \\
\hline I & $\llbracket x^{2} / 10 \rrbracket$ & 4 & $1.13 e-482$ & 5.0 & $1.18 e-388$ & 5.0 \\
\hline
\end{tabular}


Table 3. Problem 3: Initial guess: $x_{i}=15 / 10, n=3$, Iter $=8$

\begin{tabular}{|c|c|c|c|c|c|c|c|c|}
\hline \multicolumn{7}{|c|}{ Methods (16) } & \multicolumn{2}{|c|}{ Method (17) } \\
\hline Methods & $M_{1}(\mathbf{x})$ & $M_{2}(\mathbf{x})$ & $\mathbf{V}(\mathbf{x})$ & $m$ & $\|\mathbf{F}(\mathbf{x})\|_{\infty}$ & $\mathrm{CCO}$ & $\|\mathbf{F}(\mathbf{x})\|_{\infty}$ & $\mathrm{CCO}$ \\
\hline (7) & $\mathbf{R}_{1}$ & I & - & 1 & $1.63 e-241$ & 2.0 & $6.69 e-268$ & 2.0 \\
\hline (7) & I & $\mathbf{R}_{1}$ & - & 1 & $1.34 e-281$ & 2.0 & $6.69 e-268$ & 2.0 \\
\hline (7) & $\mathbf{R}_{1}$ & I & - & 2 & $1.19 e-6427$ & 3.0 & $1.74 e-6229$ & 3.0 \\
\hline (7) & I & $\mathbf{R}_{1}$ & - & 2 & $4.27 e-8197$ & 3.0 & $1.74 e-6229$ & 3.0 \\
\hline (13) & I & I & $\mathbf{R}_{2}$ & 1 & $1.49 e-272$ & 2.0 & $6.69 e-268$ & 2.0 \\
\hline (14) & $\llbracket \mathbf{F}(\mathbf{x}) \rrbracket$ & I & $0.001 \mathbf{R}_{2}$ & 1 & $4.43 e-290$ & 2.0 & $6.69 e-268$ & 2.0 \\
\hline (14) & $\llbracket \mathbf{F}(\mathbf{x}) \rrbracket$ & $\mathbf{I}$ & $0.001 \mathbf{R}_{3}$ & 1 & $2.22 e-283$ & 2.0 & $6.69 e-268$ & 2.0 \\
\hline (14) & $\llbracket \mathbf{F}(\mathbf{x}) \rrbracket$ & I & $0.001 \mathbf{R}_{4}$ & 1 & $1.25 e-286$ & 2.0 & $6.69 e-268$ & 2.0 \\
\hline$(14)$ & I & $\llbracket \mathbf{F}(\mathbf{x}) \rrbracket$ & $0.001 \mathbf{R}_{2}$ & 1 & $2.84 e-270$ & 2.0 & $6.69 e-268$ & 2.0 \\
\hline (14) & $\mathbf{I}$ & $\llbracket \mathbf{F}(\mathbf{x}) \rrbracket$ & $0.001 \mathbf{R}_{3}$ & 1 & $3.11 e-270$ & 2.0 & $6.69 e-268$ & 2.0 \\
\hline (14) & I & $\llbracket \mathbf{F}(\mathbf{x}) \rrbracket$ & $0.001 \mathbf{R}_{4}$ & 1 & $5.34 e-273$ & 2.0 & $6.69 e-268$ & 2.0 \\
\hline (15) & $\llbracket-\mathbf{F}(\mathbf{x}) \rrbracket$ & I & $\mathbf{R}_{5}$ & 1 & $1.36 e-278$ & 2.0 & $6.69 e-268$ & 2.0 \\
\hline (15) & I & I & $\mathbf{R}_{6}$ & 1 & $3.74 e-296$ & 2.0 & $6.69 e-268$ & 2.0 \\
\hline (15) & I & I & $\mathbf{R}_{7}$ & 1 & $4.75 e-300$ & 2.0 & $6.69 e-268$ & 2.0 \\
\hline (15) & $\mathbf{I}$ & $\mathbf{I}$ & $\mathbf{R}_{8}$ & 1 & $3.73 e-334$ & 2.0 & $6.69 e-268$ & 2.0 \\
\hline (15) & I & I & $\mathbf{R}_{9}$ & 1 & $7.06 e-325$ & 2.0 & $6.69 e-268$ & 2.0 \\
\hline (15) & $\mathbf{I}$ & I & $\mathbf{R}_{10}$ & 1 & $5.79 e-303$ & 2.0 & $6.69 e-268$ & 2.0 \\
\hline \multicolumn{9}{|c|}{$\mathbf{R}_{1}=(0.1 \exp (0.1 \mathbf{x}))(0.1 \exp (0.1 \mathbf{x}))^{T}, \mathbf{R}_{2}=[1,0,0]^{T}, \mathbf{R}_{3}=[0,1,0]^{T}, \mathbf{R}_{4}=[0,0,1]^{T}$} \\
\hline \multicolumn{9}{|c|}{$\mathbf{R}_{5}=0.001[-1,-1,-1]^{T}, \mathbf{R}_{6}=0.001[1,-1,1]^{T}, \mathbf{R}_{7}=0.001[-1,-1,1]^{T}, \mathbf{R}_{8}=0.001[-1,-1,2]^{T}$} \\
\hline \multicolumn{9}{|c|}{$\mathbf{R}_{9}=0.001[-1,-2,1]^{T}, \mathbf{R}_{10}=0.001[-2,-1,1]^{T}$} \\
\hline
\end{tabular}




\section{References}

1. J. M. Ortega, W. C. Rheinbodt, Iterative solution of nonlinear equations in several variables, United Kingdom: Academic press limited 24-28 Oval road, London NW1 7DX, 1970.

2. J. F. Traub, Iterative Methods for the Solution of Equations, Prentice-Hall, Englewood Cliffs, 1964.

3. R. L. Burden, J.D. Faires, Numerical Analysis. PWS Publishing Company, Boston, USA, 2001.

4. J. M. McNamee, Numerical Methods for Roots of Polynomials. Part I, Elsevier, Amsterdam, Holland, 2007.

5. M. Z. Ullah, F. Soleymani, A. S. Al-Fhaid, Numerical solution of nonlinear systems by a general class of iterative methods with application to nonlinear PDEs, Numerical Algorithms, vol. 67, pp. 223-242, 2014.

6. H. Montazeri, F. Soleymani, S. Shateyi, S.S. Motsa, On a New Method for Computing the Numerical Solution of Systems of Nonlinear Equations, Journal of Applied Mathematics, vol. 2012, Article ID 751975, 15 pages, 2012, doi:10.1155/2012/751975.

7. A. Cordero, M. Kansal, V. Kanwar, A stable class of improved second-derivative free Chebyshev-Halley type methods with optimal eighth order convergence ,Numer. Algor. 72, 937. 2016.

8. V. Arroyo, A. Cordero, J. R. Torregrosa, Approximation of artificial satellites' preliminary orbits: The efficiency challenge, Math. Comp. Model. 54(7-8), 1802-1807, 2011.

9. D. A. Budzko, A. Cordero, J. R. Torregrosa, New family of iterative methods based on the Ermakov-Kalitkin scheme for solving nonlinear systems of equations, Comput. Math. and Math. Phys. 55, 2015.

10. S. Qasim, Z. Ali, F. Ahmad, S. S-Capizzano, M. Z. Ullah, A. Mahmood, Solving systems of nonlinear equations when the nonlinearity is expensive, Comput. Math. Appl. 71(7), 1464-1478, 2016.

11. U. Qasim, Z. Ali, F. Ahmad, S. S-Capizzano, M. Z. Ullah, M. Asma, Constructing Frozen Jacobian Iterative Methods for Solving Systems of Nonlinear Equations, Associated with ODEs and PDEs Using the Homotopy Method, Algo. 9(1), 2016.

12. A. Cordero, J. L. Hueso, E. Martinez, J.R. Torregrosa, A modified Newton-Jarratt's composition, Numerical Algorithms, vol. 55, pp. 87-99, 2010.

13. F. Ahmad, E. Tohidi, J. A. Carrasco, A parameterized multi-step Newton method for solving systems of nonlinear equations, Numerical Algorithms, pp. 1017-1398, 2015.

14. M. Z. Ullah, S. Serra-Capizzano, F. Ahmad, An efficient multi-step iterative method for computing the numerical solution of systems of nonlinear equations associated with ODEs, Applied Mathematics and Computation, vol. 250, pp. 249-259, 2015.

15. F. Ahmad, E. Tohidi, M. Z. Ullah, J. A. Carrasco, Higher order multi-step Jarratt-like method for solving systems of nonlinear equations: Application to PDEs and ODEs, Computers \& Mathematics with Applications, vol. 70, no. 4, pp. 624-636, 2015, http://dx.doi.org/10.1016/j.camwa.2015.05.012.

16. X. Wu, Note on the improvement of Newton's method for systems of nonlinear equations, Applied Mathematics and Computation, vol. 189, pp. 1476-479, 2007.

17. J. L. Hueso, E. Martínez, J. R. Torregrosa, Modified Newton's method for systems of nonlinear equations with singular Jacobian, Journal of Computational and Applied Mathematics, vol. 224, pp. 77-83, 2009.

18. M. A. Noor and F. A. Shah, A Family of Iterative Schemes for Finding Zeros of Nonlinear Equations having Unknown Multiplicity, Appl. Math. Inf. Sci., vol. 8, no. 5, pp. 2367-2373, 2014.

19. M.A. Noor, M. Waseem, K.I. Noor, E. Al-Said, Variational iteration technique for solving a system of nonlinear equations, Optim. Lett., vol. 7, pp. 991-1007, 2013,doi 10.1007/s11590-012-0479-3.

(C) 2017 by the authors; licensee Preprints, Basel, Switzerland. This article is an open access article distributed under the terms and conditions of the Creative Commons Attribution (CC-BY) license (http://creativecommons.org/licenses/by/4.0/). 